\title{
Implant-retained overdentures did not have a significant improvement in dietary intake
}

\author{
Abstracted from \\ Hamdan NM, Gray-Donald K, Awad MA, Johnson-Down L, Wollin S, Feine JS. \\ Do implant overdentures improve dietary intake? A randomized clinical trial. J Dent Res 2013; 92: 146S-53S. \\ doi: 10.1177/0022034513504948. Epub 2013 Oct 24. PubMed PMID: 24158335; PubMed Central PMCID: PMC3860059. \\ Address for correspondence: Jocelyne Feine, Faculty of Dentistry, McGill University, Montreal, Canada. \\ E-mail: jocelyne.feine@mcgill.ca
}

\section{Question: Do implant overdentures improve the nutritional status of edentate elders?}

\section{Design Randomised controlled trial}

Intervention Patients aged 65 or above edentate for a minimum of five years, with sufficient bone for two implants in the anterior mandible, were recruited. Those with systemic disease that contraindicated implants or had a low mini-mental state evaluation score were excluded. Patients in both groups had a standard maxillary complete denture fabricated. Patients randomised to the treatment group received a two-implant mandibular overdenture while those in the control group received a standard mandibular complete denture. Three 24-hour dietary recalls were collected by telephone interviews at baseline and at 12 months. Results 255 patients were randomised: 128 received a standard complete denture (CD) and 127 a two-implant mandibular overdenture (IOD). 127 patients were available at 12-month follow up, 114 in the CD group and 103 in the IOD group. No significant between-group differences were found. Conclusions Although there is much evidence supporting the adoption of two-implant mandibular overdenture (IOD) treatment as the standard of care for edentate patients, this evidence does not include an improvement in dietary intake at one year for medically healthy independent edentate elders when given no specific dietary counselling.

\section{Commentary}

In dentistry, in addition to simply an ageing population, clinicians have observed significant changes in the oral health of older adults. Whilst this has resulted in a significant decrease in the numbers of edentulous older adults, their clinical management is becoming increasingly complex. ${ }^{1}$ Oral rehabilitation with implant-supported prostheses has become a well-documented therapy for edentate adults, particularly in the lower arch. Numerous studies have proven the benefits of such treatment for edentulous patients including improvements in Oral Health-related Quality of Life and masticatory function. ${ }^{2}$

Based on a sample size calculation, 255 independently living edentate adults aged 65 years and older were recruited in Montreal, Canada. The participants were randomly assigned to two treatment groups using block randomisation, where patients received either a complete conventional lower denture or a two-implant retained lower overdenture. In both groups patients were provided with a conventional maxillary complete denture. The outcome measure reported was the impact of prosthodontic rehabilitation on dietary intake. Patients self-reported food intake using a 24 dietary recall method at baseline and again 12 months after treatment intervention. Dietary intake values were used to calculate intake of dietary fibre, macronutrients (proteins, fat and carbohydrates), micronutrients (vitamins $\mathrm{A}$, B6, B12, C and D, thiamine, riboflavin, folate and niacin) and energy. Statistical analysis revealed no significant between-group differences in terms of intake of dietary fibre $(\mathrm{p}=0.36)$, energy $(\mathrm{p}=0.58)$, macronutrients $(\mathrm{p}=0.41)$ or micronutrients $(\mathrm{p}=0.13)$ at either baseline or 12 months after prosthodontic intervention.

Numerous studies have shown that as natural teeth are lost, chewing function can be negatively affected. This in turn is associated with negative effects on dietary choice and nutritional status. ${ }^{3}$ The results of this study mirror others which suggest that prosthodontic rehabilitation alone is insufficient to improve dietary intake amongst older patients. ${ }^{4}$ Whilst the limitations of a self-reported measure of dietary intake are discussed by the authors, the progressive change in dietary habits resulting from a declining masticatory performance over a period of years in this patient group must not be underestimated. As acknowledged in this article, such a change was unlikely to be reversed by simply replacing missing teeth, and in the absence of targeted dietary advice was insufficient to attain a significant improvement in dietary intake for this study population.

\section{Practice points}

- Implant-retained overdentures have been shown to have very positive impacts on patients' Oral Health-related Quality of Life and masticatory function

- In this study, provision of implant-retained overdentures did not result in a significant improvement in dietary intake compared to conventional removable dentures.

\section{Gerald McKenna}

Cork University Dental School and Hospital, University College Cork, Ireland

1. Thomason JM, Kelly SA, Bendkowski A, Ellis JS. Two implant retained overdentures a review of the literature supporting the McGill and York consensus statements. J Dent 2012; 40: 22-34.

2. Awad MA, Rashid F, Feine JS. The effect of mandibular 2-implant overdentures on oral health-related quality of life: an international multicentre study. Clin Oral Implants Res 2014; 25: 46-51.

3. Hyland R, Ellis JS, Thomason MJ, El-Feky A, Moynihan P. A qualitative study on patient perspectives of how conventional and implant-supported dentures affect eating. J Dent 2009; 37: 718-723.

4. Wostmann B, Michel K, Brinkert, Melchheier-Weskott A, Rehmann P, Balkenhol M. Influence of denture improvement on the nutritional status and quality of life of geriatric patients. J Dent 2008; 36: 816-821.

Evidence-Based Dentistry (2014) 15, 89. doi:10.1038/sj.ebd.6401048 